\title{
Recent Trends on Content Based Image Retrieval System- An Overview
}

\author{
Rajsheel Sharma ${ }^{1}$,Prof. Ratnesh Dubey ${ }^{2}$, Dr.Vineet Richariya ${ }^{3}$ \\ ${ }^{I}$ (M.Tech Scholor, LNCT, Bhopal) \\ ${ }^{2}$ (Asst. Professor, CSE Department, LNCT, Bhopal) \\ ${ }^{3}$ (HOD,CSE Department, LNCT ,Bhopal)
}

\begin{abstract}
The Content-Based Image Retrieval (CBIR) techniques comprise methodologies intended to retrieve self-content descriptors over the image data set being studied according to the type of the image. The main purpose of CBIR consists in classifying images avoiding the use of manual labels related to understanding of the image by the human being vision. This paper provides an overview on Recent Trends of CBIR includes the Relevance Feedback (RF), Interactive Genetic Algorithm, Neural Network etc.. Relevance Feedback enhances the ability of CBIR effectively by dropping the semantic gap between low-level features and high level features. Interactive Genetic Algorithm is a branch of evolutionary computation which makes the retrieval process more interactive so that user can get advanced results from database matching to Query Image with his evaluation. Neuro-fuzzy logic based implicit feedback gets improved results as compared to traditional implicit feedback.
\end{abstract}

Keywords : CBIR, Neuro-fuzzy logic, Relevance Feedback, Interactive Genetic Algorithm, Image Retrieval (IR).

\section{Introduction}

To reduce the lack of regularity difficulty, the image retrieval is carried out according to the image features. Such scheme is the so-called content-based image retrieval (CBIR). The main challenge of the CBIR system is to construct having important effect descriptions of physical attributes from images to accelerate efficient and effective retrieval. CBIR has become an energetic and fast- improving research area in image retrieval in the last few years. Due to this CBIR have improved in lots of way such as region-level features based, relevance feedback, semantic based etc.

We often need to efficiently store and retrieve image data to perform assigned tasks and to make a decision. Therefore, developing proper tools for the image retrieval on the basis of image content from large image database is challenging. Generally there are two different types of approaches (1) text based retrieval (2) content based retrieval, are usually adopted in image retrieval. In the text-based system, the images are manually annotated by text descriptors and then used by a database management system to perform image retrieval. However, there are two limitations of using keywords to achieve image retrieval: the large works have to do for manual image annotation and the task of describing image content is highly subjective. The perspective of textual descriptions given by an annotator could be different from the perspective of a user. In other words, there are inconsistencies between user textual queries and image annotations or descriptions. The image retrieval is carried out according to the image contents. This technique is so-called content-based image retrieval (CBIR). The primary goal of the CBIR system is to construct meaningful descriptions of physical attributes from images to facilitate efficient and effective retrieval $[2,3]$.

Content based features are chiefly divided into two main region; Common visual features and Field Specific visual features such as face recognition, task dependent applications etc. On the other hand, high level features include semantic based image retrieval computed from text description or by complex algorithms of visual features. The combination of these content based features is required for improved retrieval of image according to the application. These are the features of the image [1].

CBIR has become an active and fast-advancing research area in image retrieval in the last decade. A huge research activities in CBIR have progressed in following areas (1) region-level features based (2) global image properties based (3) relevance feedback and (4) semantic based image retrieval .There are different approach of CBIR algorithms has been proposed, but most of them focus on the similarity computation phase to find effectively and efficiently a desired image or a group of images that are similar to the given query. To achieve better results of the user's choice information need for the following search in the image database, involving user's interaction is necessary for a CBIR system. 


\section{Content Based Image Retrival: Overview}

Fig. 1 shows a generic description of a standard image retrieval system.

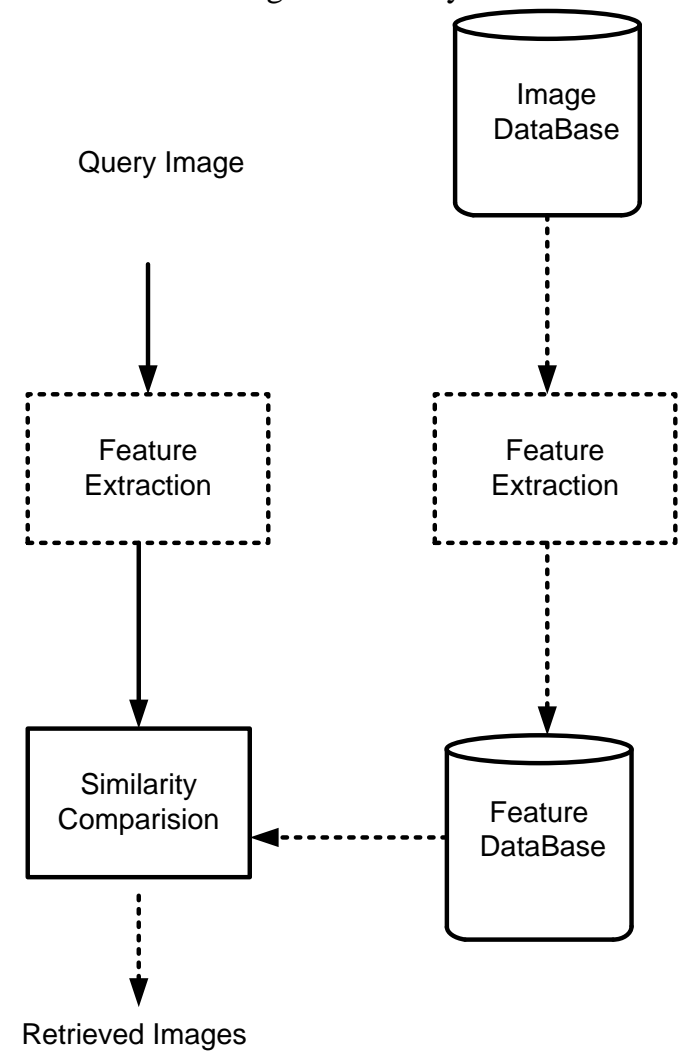

Fig. 1: CBIR System

Any CBIR system involves at least three following steps: -

- Feature extraction and indexing of image database according to the chosen visual feature color, shape, texture or any combination of the above.

- Feature extraction of query image(s).

- Matching the query image to the most similar images in the database. This forms the search part of the CBIR [6].

\section{CBIR Terminology}

Color: Color is a leading and visible feature for image retrieval. Typically CBIR systems use color space, histogram, moments, color coherence vector and dominant color descriptor to signify color.

Texture: Texture quality is described as a information of local shape and color feature or in a additional descriptive way it is called as structure and randomness. Structural schemes contain graphical technique which measured to be more resourceful when applied to the texture. Randomness methods symbolize Tamura features, Markov random field, wavelet transform, dual tree complex wavelet and contour lets. Texture can be represented by Grey Level Co-occurrence matrix. Texture is an essential quality for general images but its comprehensive definition does not exist still yet.

Edge: Edge finding defined as to the method of inspection and locating sharp indefinite in an image. This indefinite or discontinuities are discriminate as boundaries of objects in a scene that is rapid changes in pixel intensity. Typically Classical edge detection schemes occupy the image with an operator ( 2-D filter), which is constructed to be influence to large gradient in the image while inveterate values of zero in uniform regions. There are lot of edge detection operators existing, each design to be sensitive to certain types of edges. Variables involved in the selection of an edge detection operator include:

- Edge orientation: The geometry of the operator determines a characteristic way in which it is most responsive to edges. Operators can be optimized to look for parallel, perpendicular, or sloping edges.

- Noise environment: Edge detection is difficult in noisy images, hence both the noise and the edges having 
high frequency content. Attempts to reduce the noise result in blurred and distorted edges. Operators used on noisy images are typically larger in scope, so they can average enough data to discount localized noisy pixels. This results in less accurate localization of the detected edges [2].

Relevance Feedback:-The difference between the user's information need and the image representation is called the semantic gap in CBIR systems. The limited retrieval accuracy of image nuclear retrieval systems is essentially due to the intrinsic semantic gap. In order to reduce the gap, relevance feedback is very helpful into CBIR system.

The basic idea behind relevance feedback is to integrate human perception subjectivity into the query and involve user to evaluate the retrieval results. Then depending upon user's integration the similarity measures are automatically refined. There are lots of CBIR algorithms has been proposed and most of them work on the finding effectively specific image or group of relevant image to that query image using similarity computation phase. But it is necessary to have user's interaction to get better results. Thus in order to achieve a better approximation of the user's information need for the following search in the image database, involving user's interaction is necessary for a CBIR system [3].

Interactive Genetic Algorithm:-GAs within the field of evolutionary computation, are robust, computational, and stochastic search procedures modelled on the mechanics of natural genetic systems. In general, a GA contains a fixed-size population of potential solutions over the search space. These potential solutions of the search space are encoded as binary or floating-point strings, called chromosomes. The initial population can be created randomly or based on the problem- specific knowledge.

IGA is a branch of evolutionary computation. The main difference between IGA and GA is the construction of the fitness function, i.e., the fitness is determined by the user's evaluation and not by the predefined mathematical formula. A user can interactively determine which members of the population will reproduce, and IGA automatically generates the next generation of content based on the user's input. Through repeated rounds of content generation and fitness assignment, IGA enables unique content to evolve that suits the user's preferences. Based on this reason, IGA can be used to solve problems that are difficult or impossible to formulate a computational fitness function, for example, evolving images, music, various artistic designs, and forms to fit a user's aesthetic preferences

Neuro-fuzzy logic:- Neuro-fuzzy inference system implements fuzzy inference system in the framework of Adaptive networks. NFIS is a feed forward neural network, in which the parameters of the square nodes need learning. The learning of fuzzy inference system is the adjustment about the antecedent parameters and consequent parameters [4].

\section{ADVANTAGES OF CBIR}

- Easy to implement.

- Fast retrieval.

- Web image search (surrounding text).

- Manual annotation is not always available.

\section{Disadvantages Of CBIR}

- Manual annotation is impossible for a large DB.

- Manual annotation is not accurate.

- A picture is worth a thousand words.

- Surrounding text may not describe the image.

\section{Application Of CBIR}

- Crime prevention: Automatic face recognition systems, used by police forces.

- Security Check: Finger print or retina scanning for access privileges.

- Medical Diagnosis: Using CBIR in a medical database of medical images to aid diagnosis by identifying similar past cases.

- Intellectual Property: Trademark image registration, where a new candidate mark is compared with existing marks to ensure no risk of confusing property ownership.

- Architectural and engineering design Designer needs to be aware of previous designs, particularly if these can be adapted to the problem at hand. Hence the ability to search design archives for previous examples which are in some way similar, or meet specified suitability criteria, can be valuable. 


\section{Related Work}

Hiremath, P.S. Pujari, J. refers to the visual patterns that have properties of homogeneity or arrangement that do not result from the presence of only a single color or intensity. Various texture representations have been investigated in pattern recognition and computer vision. Two classes of texture representation methods can be distinguished: structural and statistical. Structural methods, including morphological operator and adjacency graph, describe texture by identifying structural primitives and their placement rules. They deal with the arrangement of image primitives, for instance, presence of parallel or regularly spaced objects. On the other hand, statistical methods, which include the popular co-occurrence matrix, Fourier power spectra, shift invariant principal component analysis (SPCA), Tamura feature, multiresolution filtering techniques such as Gabor and wavelet transform, characterize texture by the statistical distribution of the image intensity. The comparison of images in the context of image retrieval using texture feature is ultimately linked to the representation employed for the texture [5].

Mianchu Chen, Ping Fu, Yuan sun, Hui zhang proposed the Color represents one of the most widely used visual features in CBIR systems. First a color space is used to represent color images. Typically, RGB (red Green Blue) space, where the grey level intensity is represented as the sum of red, green and blue grey level intensities, is widely used in practice. Next, a histogram, In RGB space, one histogram for each basic color is needed-, is employed to represent the distributions of colors in image. [7]

Chin-Chin Lai et.al. have proposed an interactive genetic algorithm (IGA) to reduce the gap between the retrieval results and the users' expectation .They have used Color attributes like the mean value, standard deviation, and image bitmap .They have also used texture features like the entropy based on the gray level cooccurrence matrix and the edge histogram.[10]

Sung-Bae Cho and Joo-Young Lee had proposed a Human -Oriented Image Retrieval System to extract the feature from images by wavelet-transform and provide a user friendly means to retrieve an image from a large database when the user cannot clearly define what the image must be [12].

Linying Jiang et.al have proposed CBIR algorithm oriented by Users Experience in order to improve the storage competence, retrieval speed and accurateness of the existing CBIR algorithm as well as to improve the quality of user experience[13].

N.Srikrishna, K.Vindhya and P.Satyanarayana propose a neuro-fuzzy technique for CBIR. The CBIR technique is based on fuzzy interpretation of natural languages neural network learning and searching algorithms. Initially, fuzzy logic is developed to interpret natural expression like most, many and few. In other hand, a neural network is calculated to learn the meaning of most reddish, many reddish and few reddish. The neural network is self determining to the database used, which avoids retraining of the neural network. Lastly, a binary search algorithm is used to competition and display neural network's output and images from database. The future techniques are very exceptional and the modernization of this research is not only based on hybrid approach to CBIR but also on the fresh idea of training neural network on queries. One of the unique aspect of that research is the neural network is design to learn query and not databases. The technique can be used for any real-world online database [14].

Ganar, A.N. Gode and C.S. Jhambulkar proposed Shape extraction, which includes edges, contours, becomes an established field in itself in image processing community and various methods have been put forward. Typically, one distinguishes two main streams in this respect: methods based on quantifying edge strength using gradient like method, then looking for its local directional maxima, and methods based on search for zero-crossing in a second order derivative expression, e.g., Laplacian-like approach [15].

Ritendra Datta, Jia Li James and Z. Wang have proposed the last decade has witnessed huge interest in research on CBIR. This has covered the method for a large number of fresh techniques, and a mounting interest in associated fields to support such systems. Correspondingly, digital imagery has expanded its prospect in many directions, resultant in an detonation in the volume of image data necessary to be organize. They discuss some of the main contributions in the current decade related to image retrieval (IR) and automated image annotation. They are also discuss few of the key challenges involved in the adaptation of existing image retrieval techniques to build useful systems that can handle real world data [16].

Reshma Chaudhari and A. M. Patil have proposed CBIR uses the visual contents of an image for example shape, color, and spatial layout to represent and index the image. Active research in CBIR- is geared towards the growth of method for analyzing, interpret classification and indexing image database. In adding to their growth, efforts are also being made to assess the act of IR systems. The quality of response is heavily reliant on the choice of the method used to produce feature vectors and parallel measure for evaluation of features. They found an algorithm which incorporates the compensation of various other algorithms to get better exactness and performance of retrieval. The exactness of color histogram based matching can be increased by using Color Coherence Vector (CCV) for successive alteration. The speed of shape based retrieval can be enhanced by considering comparatively truthful shape rather than the exact shape. In addition to this a combination of color and shape based retrieval is also included to get better the exactness of the result[17]. 
Manimala Singha and K. Hemachandran have proposed Content Based Image Retrieval Technique can be found in a number of dissimilar domains such as Remote Sensing and Earth Resources Management. The CBIR, using features like shape, texture and color, called Wavelet Based Color Histogram Image Retrieval. The texture and color features are extracting through wavelet transformation and color histogram and grouping of these features is robust to scaling and translation of objects in an image. The proposed system has established a talented and faster retrieval method on a WANG image database containing 1500 general-purpose color images. The act has been evaluated by evaluating with the existing systems in the literature [18].

A. Ramesh Kumar and D. Saravanan Content-based image retrieval (CBIR) scheme searches the mostsimilar images of a query image that involve in evaluate the feature vectors of all the images in the database with that of the query image using some pre-selected similarity measure, and then sorting of the results. On query an image a reduced set of candidate images which have similar Grid Code as that of the query image is obtain. The color histogram for an image is constructed by quantizing the colors within the image and counting the number of pixels of each color. The feature vector of an image can be derived from the histograms of its color components and finally can set the number of bins in the color histogram to obtain the feature vector of desired size. Thus the grid code of an image is obtained through the quantization of the feature vector derived from the histogram of the desired color component of the image. In order to have similar features of the images the grid code must be same for all Images in the grid. Experimental result show confirms that the proposed method is most effective [19].

Simardeep Kaur and Dr. V.K. Banga Proposed the CBIR is an lively research field in past year. Against the conventional system where images are retrieved based on the keyword search, Content Based Image Retrieval system retrieves the images based on the visual content. The presentation of HSV color space is evaluating on the basis of exactness, accuracy and Recall. We present HSV based color space image retrieval method based on the color distribution of the images [20].

R. Priyatharshini, Surendernath S.P and Dr. S. Chitrakala have proposed computer technology and World Wide Web (WWW) there has been a detonation in the amount and complexity of multimedia data that are generated, stored, transmitted, and accessed. In systematize to extract useful information from this massive amount of data, many CBIR systems have been developed in the last past year. A typical CBIR system captures image features that represent image properties such as color, texture, or shape of objects in the uncertainty image and aim to retrieve images from the database with similar features. Retrieval efficiency and accuracy are the key issues in designing CBIR System. The Shape and Spatial features are quiet easy to derive and effective. Researchers are moving towards finding spatial features and the scope of implementing these features in to the image retrieval framework for reducing the semantic gap [21].

\section{Conclusions}

Network and development of multimedia technologies are attractive more popular, users are not satisfied with the established information retrieval techniques. So now days the CBIR are becoming a source of exact and fast retrieval. There are various applications of CBIR in field of medicine like dermatology, blood cell detection etc. Thus, field of CBIR is very useful for practical reasons. The purpose of this survey is to provide an overview of the functionality of content based image retrieval systems. Most systems use color and texture features, few systems use shape feature, and still less use layout features. With the aid of genetic algorithm based similarity measure and use of feedback techniques, images that are similar to the query image are retrieving effectively and efficient. Use of genetic algorithm in CBIR system reduces the computation time. This new approach reduce "semantic gap" between the human perception and visual content and increase performance of system.

\section{References}

[1] Gudivada V. N., Raghavan V. V., "Content based image retrieval systems," IEEE Computer,. 28, pp. 18-22,1995

[2] Yong-Rui Thomas S., Huang, Michael Ortega, and Sharad Mehrotra" Relevance Feedback: A Power Tool for Interactive ContentBased Image Retrieval” IEEE Trans. on Circuits and Systems For Video Technology, Vol. 8, No.5, pp.664-665 sep. 1998.

[3] Ding, W-Zijun Yang, Jay-Kuo, "Survey on content-based analysis, indexing and retrieval techniques and status report of MPEG-7", journal of science and engineering, Vol. 2, No. 3 pp. 101-118, July 1999.

[4] X. S. Zhou and T. S. Huang, "Relevance feedback in CBIR Some recent advances" Inf. Sci., vol. 148, no. 1-4, pp. 129-137, Dec. 2002.

[5] Hiremath, P.S.Pujari "Content based image retrieval using color, texture and shape features," Advanced Computing and Communications, International Conference on , pp.780-784, Dec. 2007

[6] Anelia Grigorova, Francesco G. B. De Natale, Charlie Dagli, and Thomas S. Huang, "Content-Based Image Retrieval by Feature Adaptation and Relevance Feedback” IEEE conference on Multimedia, Vol. 9, No. 6, pp. 1183-1192 ,Oct.2007.

[7] Mianchu Chen, Ping Fu-Yuan sun, Hui-zhang "Image Retrieval Based on Multi-feature similarity score fusion using Genetic Algorithm" The 2nd International Conference on Computer and Automation Engineering (ICCAE), vol. 6, pp no. 751-759, Feb. 2010.

[8] Lijun Zhao and Jiakui Tang, " CBIR Using Optimal Feature Combination and Relevance Feedback", 2010 International Conference on Computer Application and System Modeling (ICCASM 2010) vol 8 pp no. 624-627 March 2010. 
[9] Ching Hung Su, Huang Sen Chiu and Tsai Ming Hsieh, "An efficient image retrieval based on HSV color space," Electrical and Control Engineering (ICECE), 2011 International Conference on , pp.5746-5749, 16-18 Sep. 2011.

[10] Chih-Chin Lai, "A User-Oriented Image Retrieval System Based on Interactive Genetic Algorithm, "IEEE TRANSACTIONS ON INSTRUMENTATION AND MEASUREMENT/0018-9456/2011.

[11] Raghupathi Gali, M.L.Dewal, R.S.Anand," Genetic algorithm for content based image retrieval" 4th International Conference on Computation Intelligence, Communication Systems and Networks, July 2012.

[12] S.B. Cho and J.Y. Lee, "A human-oriented image retrieval (IR) system using interactive genetic algorithm," IEEE Trans. Syst., Man, Cybern. System Humans, vol. 32, no. 3, pp. 452-458, May 2002.

[13] Linying Jiang, Jiefu Ren and Dancheng Li,"CBIR algorithm oriented by User's Experience", 7th International Conference on Computer Science \& Education (ICCSE 2012) ,July 14-17, 2012. Melbourne, Australia.

[14] N.Srikrishna, K.Vindhya and P. Satyanarayana," A neuro fuzzy approach to CBIR," 7th Conference on Global spatial data infrastructure, Aug. 2004.

[15] Ganar, A.N. Gode, C.S. Jambhulkar and S.M. "Enhancement of Image Retrieval by Using Color, Texture and Shape Features" IEEE conference on Multimedia, Vol. 9, No. 6, pp. 1212-1216,Oct.2007

[16] Ritendra Datta, Jia Li James and Z - Wang, "CBIR- Approaches and Trends of the New Age" 4th International Conference the Next Generation Information Technology Summit, vol. 7 pp. 721-726, sep. 2013.

[17] Reshma Chaudhari and A. M. Patil "Content Based Image Retrieval Using Color and Shape Features" International Journal of Advanced Research in Electrical, Electronics and Instrumentation Engineering Vol. 1, Issue 5, November 2012

[18] M. Singha and K. H. chandran "CBIR using Color and Texture"Signal \& Image Processing : An International Journal (SIPIJ) Vol.3, No.1, Feb. 2012

[19] A. Ramesh Kumar and D. Saravanan "Content Based Image Retrieval Using Color Histogram" (IJCSIT) International Journal of Computer Science and Information Technologies, Vol. 4 (2), 2013, pp $242-245,2013$.

[20] Simardeep Kaur and Dr. V. K. Banga "CBI R: Survey and Comparison between RGB and HSV model" International Journal of Engineering Trends and Technology - Volume4 Issue4 - Apr. 2013.

[21] R. Priyatharshini, Surendernath S.P and Dr. S. Chitrakala "Application of Spatial Features in CBIR: A Survey" Third International Conference on Computer Science, Engineering \& Applications (ICCSEA 2013), May 24 26, 2013, Delhi, India. 